Hautarzt 2021 · 72:563-569

https://doi.org/10.1007/s00105-021-04831-3

Angenommen: 6. Mai 2021

Online publiziert: 11. Juni 2021

(c) Der/die Autor(en) 2021

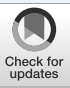

\section{Mikrobiom als natürlicher Schutzfaktor}

\author{
Perspektiven aus der Grundlagenforschung
}

\author{
Thomas C. G. Bosch \\ Zoologisches Institut, Christian-Albrechts-Universität Kiel, Kiel, Deutschland
}

\section{Zusammenfassung}

\section{In diesem Beitrag}

\section{- Mikroben neu denken \\ - Was ist ein gesundes Mikrobiom? \\ - Mikrobiome sind Filter \\ - Prinzip Kolonisierungsresistenz \\ - Mikroben sind Teil des Immunsystems \\ - Vom Mikrobiom zu den Lifestyle-Krank- heiten \\ - Die Beeinflussbarkeit des Mikrobioms \\ - Das Mikrobiom ist in akuter Gefahr}

Hintergrund: Eine neue Generation von Technologien deckt eine große Zahl von stehen. Störungen dieser Partnerschaft haben erhebliche Konsequenzen. Seit Jahrzehnten schreitet die Verarmung des Mikrobioms im Zuge eines modernen, den genetischen Aspekten auch die auf der Haut und anderen Organen lebenden Mikroben zu berücksichtigen. Alle Epithelien einschließlich der Haut sind mit einer Vielzahl von Mikroben besiedelt. Organen.

Material und Methode: Es erfolgt eine Diskussion von Grundlagenarbeiten. Mikroorganismen auf, die mit der Haut in einer engen und oft funktionellen Beziehung globalisierten Lebensstils voran. Bei der Aufrechterhaltung der Gesundheit sind neben

Fragestellung: Betrachtet wird die Funktion des Mikrobioms in der Haut und anderen

Ergebnisse: Das Mikrobiom der Haut ist für die Aufrechterhaltung der Gesundheit von großer Bedeutung.

Schlussfolgerungen: Wir brauchen das Verständnis unseres Körpers als multiorganismischer Metaorganismus, um intelligent auf die Herausforderungen einer sich immer rascher ändernden Umwelt reagieren zu können.

Schlüsselwörter

Mikrobiota $\cdot$ Symbiose $\cdot$ Lifestyle-Erkrankungen $\cdot$ Antibiotika $\cdot$ Mikroorganismen

Fortschritte in der Mikrobiomforschung haben unsere Denkweise über Gesundheit und Krankheit und unser Bild vom Menschen verändert. Menschen, ihre Organe und ihr Immunsystem funktionieren nur in Zusammenarbeit mit Mikroben. Das Metaorganismuskonzept definiert den Organismus und die mit ihm assoziierten Bakterien als Einheit. Die Störung dieses Zusammenspiels wird als Ursache für viele moderne Krankheitsbilder auch der Haut gesehen.

\section{Mikroben neu denken}

Bakterien besiedeln in großer Zahl und Vielfalt ungefähr $4 \mathrm{Mrd}$. Jahre länger die Erde als der Mensch [1-3]; $37 \%$ der menschlichen Erbsubstanz können auf bakterielle Vorfahren zurückgeführt werden; sie sind uralter Teil unseres Selbstseins [3]. Weniger als 200 Bakterienarten gelten dabei gemeinhin als ausschließlich krankheitserregend.

\section{》) Die Hautgesundheit hängt maßgeblich vom Zustand des Hautmikrobioms ab}

Bis vor Kurzem wurde den Bakterien auf unserer Haut, in unserer Mundhöhle, in den Atemwegen und im Darm kaum Beachtung geschenkt. Technologische Fortschritte in der Entschlüsselung der Erbinformation haben in den letzten Jahren eine völlig neue und weitgehend unsichtbare Welt sichtbar gemacht und gezeigt, dass sich in unseren Geweben und Organen eine Fülle von genetischen Fußabdrücken entdecken lässt, die unzählig vielen Mikroorganismen zugeordnet werden können. Es ist dadurch möglich geworden, das „Mikrobiom" einzelner Organe oder des gesamten menschlichen Körpers in einer 


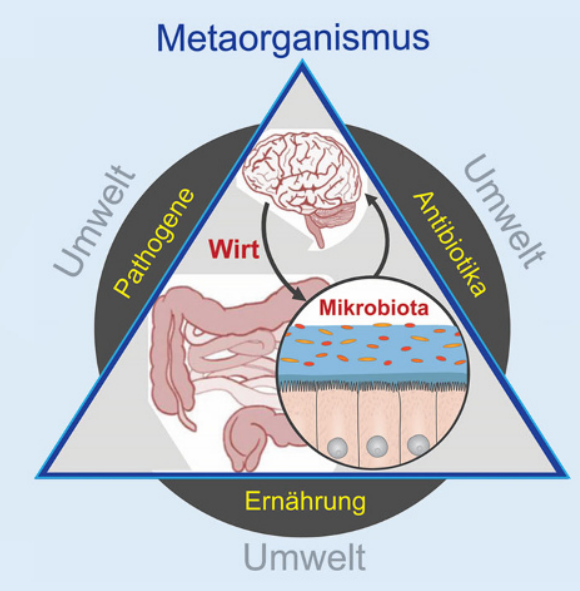

Abb. $1<$ Individuum als Metaorganismus und damit als komplexe Gemeinschaft von vielen Arten, die miteinander eng vernetzt sind. Umwelteinflüsse beeinflussen den Metaorganismus in vielfältiger Weise. (Mit freundl. Genehmigung $\odot$ K. Schröder, alle Rechte vorbehalten)

Vielzahl von kranken und gesunden $\mathrm{Zu}$ ständen zu bestimmen. Heute wissen wir $[4,5]$, dass

- die allermeisten der uns besiedelnden Mikroben keine Krankheitserreger sind, sondern dass wir sie für unsere Entwicklung und auch zum Schutz vor möglichen infektiösen Erregern brauchen,

- unsere Haut je nach Feuchtigkeit von einigen Hundert und Hunderttausenden von Bakterien pro Quadratzentimeter kolonisiert wird, die bestens angepasst sind, um die auf der Haut nur spärlich verfügbaren Nährstoffe zu nutzen,

- es zwischen unseren Organen wie der Haut, der Mundhöhle, dem Darm und auch dem Gehirn eine enge zelluläre und molekulare Verbindung zu den besiedelnden Mikroben gibt,

- eine Störung der Zusammensetzung des Hautmikrobioms mit einer Reihe von Hauterkrankungen assoziiert ist,

- Organismen immer multiorganismisch sind und es im engeren Sinn keine Individuen gibt, die für sich alleine bestehen können,

- wir nur als Ökosystem existieren in einer evolutionären Partnerschaft mit Mikroben - und wir uns daher besser als Metaorganismus oder Holobiont

(-Abb. 1) verstehen sollten.

\section{Was ist ein gesundes Mikrobiom?}

Trotz vieler Hinweise auf die Rolle des Mikrobioms für die menschliche Gesundheit ist immer noch unklar, was wir unter einem gesunden oder vorteilhaften Mikrobiom zu verstehen haben. Ein Teil des Problems sind die großen individuellen Unterschiede zwischen den Mikrobiomen scheinbar gesunder Menschen, die sich auf die komplexen Interaktionen von persönlichen Umwelt-, genetischen und Lebensstilfaktoren zurückführen lassen. Relativ kleine Unterschiede können eine unverhältnismäßig große Rolle spielen bei der Frage, ob eine Person relativ gesund ist oder ein erhöhtes Risiko hat zu erkranken. Mit anderen Worten, das gesunde Mikrobiom einer Person ist möglicherweise in einem anderen Kontext nicht gesund. Das Bild wird noch komplexer, weil bestimmte mikrobielle Konsortien sich nicht unbedingt immer aus den gleichen Mikrobenarten zusammensetzen müssen. Aufgrund der erheblichen metabolischen Redundanz sind häufig Gene mit derselben Funktion auf viele Bakterienarten verteilt. Dadurch kann ein gesundes Mikrobiom in unterschiedlichen Individuen durchaus aus unterschiedlichen Arten zusammengesetzt sein. "It's the song, not the singer ..." [6], auf den es am Ende ankommt.

Dazu kommt, dass sich die taxonomische Zusammensetzung der mikrobiellen Gemeinschaft im Laufe des Lebens verändern kann. Es kommt also nicht auf die einzelnen Arten, sondern auf ihre Funktionalität an. Genauso wie es kein perfektes oder gar "gesundes" Genom gibt, gibt es auch kein perfektes Mikrobiom. Wenn wir von einem gesunden oder "vorteilhaften“ Mikrobiom sprechen, meinen wir daher mikrobielle Konsortien, die nicht nur mit unserer Lebensweise und unserer ökologi- schen, soziokulturellen und auch ökonomischen Umwelt harmonieren, sondern auch mit unserem genetischen Hintergrund.

\section{Mikrobiome sind Filter}

Um zu begreifen, wie der Zusammenbruch dieses Ökosystems der komplexen Lebensgemeinschaft aus Mensch und Mikroben zu Krankheiten führt, müssen wir verstehen, wie die unterschiedlichen Mikroben untereinander und mit unseren Zellen interagieren. Bislang gingen die Zellbiologen davon aus, dass unsere Zellen Signale aus der unmittelbaren Umgebung direkt über ihre Zellwand empfangen und prozessieren. Dafür sind ihre Oberflächen gespickt mit Sensoren und Rezeptoren. Aus dem oben Gesagten geht nun hervor, dass die meisten Epithelien und natürlich auch unsere Hautoberfläche von einer komplexen Gemeinschaft von Bakterien besiedelt sind (- Abb. 2). Viele der Signale, die aus der Umwelt ankommen, mag es sich um UV-Strahlen, Nahrung, Temperatur, Bruchstücke und Moleküle von anderen Zellen und vieles andere mehr handeln, werden daher zunächst durch das Mikrobiom gefiltert, bevor sie die Außenmembran unserer Zellen erreichen. Das Mikrobiom dient damit als lebender und schützender Filter zwischen unseren Epithelien und der Umwelt.

\section{Prinzip Kolonisierungsresistenz}

Schon in den 1950er-Jahren wurde im Tiermodell ein Zusammenhang zwischen Dysbiose infolge einer Antibiose und einer deutlich erhöhten Suszeptibilität gegenüber bakteriellen Infektionen beschrieben [7, 8]. Vergleichende Mikrobiomanalysen von Patienten nach Antibiotikabehandlung lieferten erste Hinweise, dass der durch die Einnahme von Antibiotika verursachte mikrobielle Diversitätsverlust mit einem erhöhten Infektionsrisiko einhergeht [9]. Diese Schutzfunktion wird auch als Kolonisierungsresistenz bezeichnet. Symbiotische Mikroben können eine solche Kolonisationsresistenz durch ganz unterschiedliche Mechanismen herbeiführen. Sie können beispielsweise „passiv“ um gemeinsame Nahrungsquellen und ähnliche ökologische Nischen kompetieren. Viele kutane Mikroorganismen können 
Hier steht eine Anzeige.

黑 Springer 


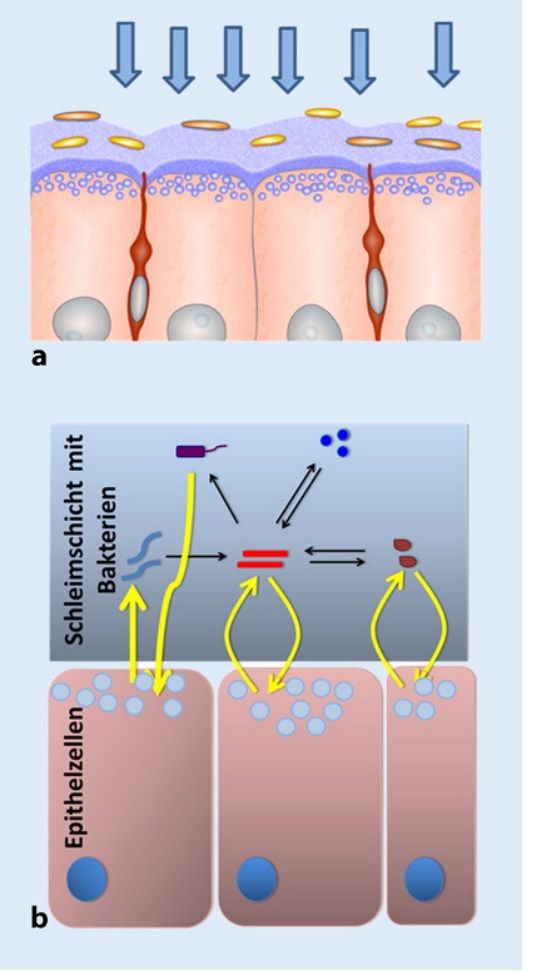

Abb. $2 \Delta$ Das Mikrobiom dient als Filter zwischen dem Wirt und der Umwelt. a Epitheliale Oberflächen wie die Haut werden von Bakterien besiedelt. Blau Schleimschicht mit Bakterien; rot Epithelzellen und dazwischenliegende Nervenzellen. Pfeile deuten Signale und Faktoren aus der Umwelt an, die zunächst auf den mikrobiellen Filter treffen (b). In der alle Epithelzellen umgebenden Schleimschicht (Mukosa) scheinen sich wahre Kriegsschlachten zwischen den Bakterien abzuspielen, begründet durch antimikrobielle Substanzen, die sowohl vom Wirt als auch von den siedelnden Bakterien wie auch durch bakterielle Kommunikationsprozesse gebildet werden. (Mit freundl. Genehmigung (c) P. Deines, alle Rechte vorbehalten)

antimikrobielle Moleküle produzieren, die das Eindringen von fremden Mikroben aus der Umgebung verhindern und die Besiedlung hemmen [10-12]. So ist seit Langem bekannt, dass eine Untergruppe von Staphylococcus-epidermidis-Stämmen die Bildung von Staphylococcusaureus-Biofilmen hemmt [13, 14]. Dies ist von Interesse, weil Staphylococcus aureus als ein signifikanter Risikofaktor für eine nachfolgende Infektion gilt [15, 16]. Von Staphylococcus lugdunensis ist bekannt, dass es das Wachstum von Staphylococcus aureus durch die Produktion des Antibiotikums Lugdunin hemmt [17, 18]. Ein weiteres Beispiel für ein Bakteri- um, das zur Kolonisierungsresistenz und zum Schutz der Haut beiträgt, ist Corynebacterium accolens. Dieses Bakterium verändert die lokale Umgebung der Haut, um das Wachstum von Streptococcus pneumoniae zu hemmen [19].

\section{》) Das Mikrobiom spielt bei bakteriellen Infektionen eine zentrale Rolle}

Unter den normalen Hautmikroben scheint sich also eine Reihe von Arten zu befinden, die entweder direkt oder über sekretierte Stoffwechselprodukte ein möglicherweise wirksameres Mittel zur Hemmung opportunistischer Krankheitserreger sein können als die herkömmlichen Antibiotika, gegen die Bakterien auch noch Resistenzen entwickeln können. Quasi als „proof of principle" für das Translationspotenzial dieser Erkenntnis kann die Beobachtung herangezogen werden, dass eine lokale Anwendung von solchen antimikrobiell wirkenden Hautmikroben die Besiedlung von Staphylococcus aureus bei Personen mit atopischer Dermatitis verringern kann [20].

\section{Mikroben sind Teil des Immunsystems}

Die mikrobiellen Mitbewohner sind ein integraler Bestandteil unserer Immunabwehr. Wir wissen seit Langem, dass keimfrei gehaltene Tiere äußerst anfällig für Pilzinfektionen sind [21]. Offensichtlich wird mit dem Entfernen der symbiotischen Bakterien auch der Schutzschild gegen Krankheitserreger wie die in der Umwelt ständig vorhandenen Pilzsporen entfernt. Das gilt nicht nur für das Tiermodell, sondern auch für den Menschen. Jeder, bei dem eine Parodontitisbehandlung mit einer oralen Antibiotikatherapie unterstützt wurde, weiß, wie schnell plötzlich ein Pilz aus der Gruppe der Hefen die Mundschleimhaut besiedeln und zu einer Kandidose führen kann.

Das gilt übrigens nicht nur für die Mikroben in der Mundhöhle, sondern auch für die Mikroben in jedem anderen unserer Organe. Mit Blick auf die Haut wird zunehmend klar, dass sich die Mikrobiomzusammensetzung von erkrankten Hautbereichen (z. B. bei atopischer Dermatitis und Psoriasis) deutlich von der gesunder
Haut unterscheidet $[18,22]$. Der Verlust von Vielfalt im Mikrobiom scheint zu einer neuen Art von Verwundbarkeit zu führen.

\section{Vom Mikrobiom zu den Lifestyle- Krankheiten}

Funktionelle Studien legen nahe, dass Änderungen in der Diversität des Mikrobioms (vornehmlich aber nicht ausschließlich des Darmmikrobioms) eng mit dem Auftreten von sog. Lifestyle-Erkrankungen assoziiert sind und zu deren Entstehen möglicherweise sogar kausal beitragen. Als Lifestyle-Erkrankungen werden Krankheiten bezeichnet, die mit der Art und Weise zusammenhängen, wie wir als Person oder als Gruppen von Menschen leben. Dazu gehören Stoffwechselerkrankungen wie Adipositas und Typ-2-Diabetes, entzündliche Darmerkrankungen, Asthma, Allergien und Immundefekte und auch eine Reihe neurogenerativer Erkrankungen, die derzeit vorher nicht gekannte Inzidenzen erreichen. Bei den meisten dieser Erkrankungen lässt sich eine deutliche Abnahme der bakteriellen Diversität beobachten, die meist auch mit dem Verlust bestimmter metabolischer Funktionen einhergeht. Die Abwesenheit der Bakterien oder auch dieStörung der normalen Bakterienzusammensetzung (Dysbiose) scheint daher mitverantwortlich zu sein für das Auftreten dieser komplexen Erkrankungen [23].

Heute wissen wir von einer Fülle von Faktoren, die die mikrobielle Kolonisierung der Haut und anderer Organe mit Mikroben positiv wie negativ beeinflussen [24-26]. Dazu gehören intensivierte Hygienepraktiken ebenso wie der übermäßige Gebrauch von Antibiotika, Alkohol und anderen Drogen sowie geänderte Geburts- und Säuglingsernährungspraktiken und nicht zuletzt die verringerte Vielfalt in der Ernährung [27].

\section{Die Beeinflussbarkeit des Mikrobioms}

Moderne Sequenziermethoden haben eine Fülle von genetisch bedingten Erkrankungen der Haut (Genodermatosen) aufgedeckt [28]. Diese Krankheiten verlaufen häufig schwer, sind aber in der Regel seltene Krankheitsbilder. Die Möglichkeiten einer entsprechenden Manipulation 
(„Editierung") des menschlichen Genoms sind Gegenstand vieler ethischer Debatten und nicht praxisrelevant. Die gleichen Sequenziertechnologien haben uns gezeigt, dass die meisten Organe von einem stabilen Mikrobiom besiedelt sind und dass die Störung oder Abwesenheit dieses Mikrobioms (Dysbiose) mit einer Reihe von chronisch inflammatorischen Erkrankungen in Zusammenhang gebracht werden kann. Viele dieser Krankheiten sind zu Volkskrankheiten geworden. Zum Beispiel hatten nach Angaben des Kinder- und Jugendgesundheitssurvey 13,2\% der Kinder in Deutschland bereits einmal in ihrem Leben ein atopisches Ekzem (Neurodermitis). Zwei Drittel der Männer (67\%) und die Hälfte der Frauen (53\%) in Deutschland sind übergewichtig; ein Viertel der Erwachsenen gilt gar als stark adipös. Auch wenn es "das" gesunde Mikrobiom nicht geben mag, sollten wir daran denken, dass unser Lebensstil reichlich Möglichkeiten bietet, das Funktionieren dieser komplexen Lebensgemeinschaft aus Mensch und Mikroben zu unterstützen -oder auch zu beeinträchtigen. Dabei ist die Aufrechterhaltung eines vorteilhaften Mikrobioms keine leichte Aufgabe: Ernährung, Medikamente, Alkohol und Drogen, aber auch körperliche Aktivität, Stress, Schlaf und auch unsere sozialen Interaktionen haben einen Einfluss auf die Zusammensetzung und Funktionalität des Mikrobioms [29]. Wie auch unsere Arbeiten im Kieler Sonderforschungsbereich „Ursprung und Funktionieren von Metaorganismen" zeigen (https://www. metaorganism-research.com/), sind $\mathrm{Mi}$ krobiome eigene Ökosysteme und bergen eine immense genetische Vielfalt und damit auch einen Reichtum an Enzymen und Stoffwechselprodukten, die der Mensch zu seinem Nutzen erschließen kann. Vieles deutet daher darauf hin, dass sich in Zukunft mit der Manipulation und Nutzung des Mikrobioms interessante Möglichkeiten für sehr gezielte therapeutische Eingriffe ergeben werden.

\section{Das Mikrobiom ist in akuter Gefahr}

Zunehmend wird klar, welch elementare Rolle die mikrobiellen Gemeinschaften für die Organismen spielen, die sie beherbergen. Umso bedauerlicher ist es, dass im Zuge unseres globalisierten Lebensstils die Verarmung des Mikrobioms seit Jahrzehnten voranschreitet. Dieser Prozess hat sich in den letzten Jahrzehnten deutlich beschleunigt und vermutlich erheblich zum Anstieg der vielen Lifestyle-Krankheiten (Fettleibigkeit, Asthma, Nahrungsmittelallergien, Herz-Kreislauf-Erkrankungen, chronische Darmentzündungen, Ösophagusreflux und Neurodermitis) beigetragen [23]. Interessanterweise nehmen all diese Krankheiten dramatisch zu, seit Antibiotika immer häufiger zum Einsatz kommen [30].

\section{I) Auch beim Einsatz von Antibiotika ist ein Umdenken dringend nötig}

Durch die antibiotikavermittelte Vernichtung der Mikroben schwindet die Vielfalt unseres Mikrobioms, was bei vielen Menschen die Widerstandsfähigkeit gegenüber Infektionen herabsetzt. Viele Bakterien auf der Haut und im Darm kehren nach einer Antibiotikabehandlung nur langsam oder gar nicht mehr zurück. Sie fehlen fortan als Teil der menschlichen Immunabwehr. Da auch die Schulung der körpereigenen Immunzellen nicht mehr richtig funktioniert, sind Allergien, Asthma, Diabetes oder chronische Darmentzündungen die Folge. Im Tiermodell wurde überzeugend nachgewiesen [30], dass schon ein kurzer, früh in der Entwicklung erfolgender Einsatz von Antibiotika zu einem Verlust der Vielfalt des Mikrobioms führt.

Aus aktuellem Anlass sei angefügt, dass natürlich auch die gegenwärtigen Maßnahmen zur Bekämpfung der Pandemie weitreichende Auswirkungen auf das menschliche Mikrobiom haben. Wir haben an anderer Stelle betont [23], dass die Implementierung von strengen Hygienepraktiken zur Eindämmung der COVID19-Übertragung jetzt absolut notwendig ist. Wir sollten aber nicht vergessen, dass eine erhöhte Hygiene auch zu mikrobiellen "Kosten" führen wird, weil die oben beschriebene Funktionalität unseres $\mathrm{Mi}$ krobioms dadurch erheblich verringert wird. Die Mikroben, die wir mit uns herumtragen, sind an vielen grundlegenden Prozessen in unserem Körper beteiligt. Diese Organismen interagieren unter an- derem mit den Immunzellen in unserer Haut und lehren sie, nur auf ernsthafte Bedrohungen zu reagieren. Im Mittelpunkt aller Bemühungen um Infektionsschutz sollte daher die Sorge um unser Immunsystem stehen und die Erkenntnis, dass ein vielseitiges Mikrobiom ein wesentlicher Teil davon ist.

\section{Fazit für die Praxis}

- Aus unserer evolutionären Perspektive wird deutlich, dass die einzigartig entwickelte Fähigkeit des Menschen, die Umwelt schnell und schneller zu verändern, zunehmend die Funktionalität unseres Mikrobioms außer Kraft setzt. Infolgedessen können von unseren Vorfahren geerbte Interaktionen zwischen den Epithelien und dem Mikrobiom nicht mehr stattfinden, und wir gefährden unsere Widerstandskraft und Gesundheit.

- Nicht der Mensch alleine, sondern das multiorganismische Kollektiv des Holobionten ist der Patient.

- Wir sollten nie vergessen, dass wir alle und schon immer in einer mikrobiellen Welt gelebt haben und dass die Mikroben einen großen Einfluss auf alle Facetten unserer Existenz haben.

\section{Glossar}

Antimikrobielle Peptide (AMP) Proteine mit kleiner Molekülmasse und antimikrobieller Breitbandaktivität. Diese Peptide sind normalerweise positiv geladen und haben sowohl eine hydrophobe als auch eine hydrophile Seite, die es dem Molekül ermöglicht, in wässriger Umgebung löslich zu sein, aber auch in lipidreiche Membranen einzutreten.

Dysbiose Ein veränderter Zustand (oder ein Ungleichgewicht) der Mikrobiota, der mit einer Änderung der Artenzusammensetzung, Häufigkeit und/oder räumlichen Verteilung verbunden ist und typischerweise mit einer Krankheit verbunden ist.

Holobiont DerWirtsorganismus und all seine symbiotischen und stabil assoziierten Mikroorganismen. Während der Begriff "Metaorganismus" eine übergeordnete Einheit definiert, die auf alle Arten von voneinander abhängigen Assoziationen anwendbar ist, ist der Begriff "Holobiont" auf bestimmte taxonomische Gruppen beschränkt.

Keimfrei Wirtsorganismen, die frei von anderen lebenden Keimen oder Mikroorganismen sind.

Metaorganismus Eine multiorganismische Assoziation, die aus einem makroskopischen Wirt und verschiedenen Mikroorganismen einschließlich Bakterien, Archaea, Pilzen, Viren besteht.

Mikroben Mikroskopisch kleine Organismen, zu denen Bakterien, Archaebakterien, Pilze und Viren gehören.

Mikrobiom Die Gesamtheit der Mikroorganismen und ihres kollektiven genetischen Materials, das in oder auf dem Körper eines makroskopischen 
Wirtsorganismus vorhanden ist. Häufig als Synonym zu Mikrobiota verwendet.

Mikrobiota Gesamtheit der Mikroorganismen, die in den verschiedenen Bereichen unseres Körpers leben und als "Barriere" zur Außenwelt dienen, von der Haut zum Darm bis zu den Atemwegen.

Symbiotische Interaktionen Eine enge und normalerweise obligatorische Verbindung zwischen 2 oder mehreren verschiedenen Organismen verschiedener Arten, die zusammenleben, oft - aber nicht notwendigerweise - zum gegenseitigen Nutzen.

Korrespondenzadresse

Prof. Dr. Dr. h.c. Thomas C. G. Bosch

Zoologisches Institut, Christian-AlbrechtsUniversität Kiel

Am Botanischen Garten 1-9, 24118 Kiel,

Deutschland

tbosch@zoologie.uni-kiel.de

Danksagung. Der Autor ist Fellow am Canadian Institute of Advanced Research (CIFAR) im Programm "Human and the Microbiome". Seine Arbeiten werden durch den DFG Sonderforschungsbereich 1182 "Ursprung und Funktionieren von Metaorganismen" an der Universität Kiel unterstützt.

\section{Einhaltung ethischer Richtlinien}

Interessenkonflikt. T.C.G. Bosch gibt an, dass kein Interessenkonflikt besteht.

Für diesen Beitrag wurden vom Autor keine Studien an Menschen oder Tieren durchgeführt. Für die aufgeführten Studien gelten die jeweils dort angegebenen ethischen Richtlinien.

Open Access. Dieser Artikel wird unter der Creative Commons Namensnennung 4.0 International Lizenz veröffentlicht, welche die Nutzung, Vervielfältigung, Bearbeitung, Verbreitung und Wiedergabe in jeglichem Medium und Format erlaubt, sofern Sie den/die ursprünglichen Autor(en) und die Quelle ordnungsgemäß nennen, einen Link zur Creative Commons Lizenz beifügen und angeben, ob Änderungen vorgenommen wurden.

Die in diesem Artikel enthaltenen Bilder und sonstiges Drittmaterial unterliegen ebenfalls der genannten Creative Commons Lizenz, sofern sich aus der Abbildungslegende nichts anderes ergibt. Sofern das betreffende Material nicht unter der genannten Creative Commons Lizenz steht und die betreffende Handlung nicht nach gesetzlichen Vorschriften erlaubt ist, ist für die oben aufgeführten Weiterverwendungen des Materials die Einwilligung des jeweiligen Rechteinhabers einzuholen.

Weitere Details zur Lizenz entnehmen Sie bitte der Lizenzinformation auf http://creativecommons.org/ licenses/by/4.0/deed.de.

\section{The skin microbiome as a natural protection factor. Insights from basic research}

Background: A new generation of technologies is uncovering a large number of microorganisms that are closely associated with the skin. Any disturbance of the interaction between skin cells and colonizing microbes has deleterious consequences. The impoverishment of the diversity of microbiome has been progressing for decades as part of a modern, globalized lifestyle. In maintaining good health, the microbes living in and on the skin and other organs must also be taken into account in addition to genetic aspects. All epithelia, including the skin, are colonized with a large number of microbes.

Objective: The function of the microbiome in the skin and other organs is described. Materials and methods: Basic research papers are discussed.

Results: The microbiome of the skin is very important for maintaining healthy skin. Conclusions: We need to understand our body as a multiorganismic metaorganism in order to be able to react intelligently to the challenges of a continually changing environment.

\section{Keywords}

Microbiota $\cdot$ Symbiosis $\cdot$ Lifestyle disease $\cdot$ Antibiotics $\cdot$ Microorganisms

\section{Literatur}

1. Bosch TCG, Miller D (2016) The Holobiont imperative: perspectives from early emerging animals. Springer, New York

2. Bosch TCG, McFall-Ngai M (2011) Metaorganisms as the new frontier. Zoology 114:185-190

3. McFall-Ngai M, Hadfield MG, Bosch TCG et al (2013) Animals in a bacterial world, a new imperative for the life sciences. Proc Natl Acad Sci USA 110:3229-3236

4. Bosch TCG (2017) Der Mensch als Holobiont - Mikroben als Schlüssel zu einem neuen Verständnis von Leben und Gesundheit. Ludwig, Kiel

5. Bosch TCG, McFall-Ngai M (2021) Animal development in the microbial world: re-thinking the conceptual framework. Curr Top Dev Biol 141:399-427

6. Doolittle WF, Booth A (2017) It's the song, not the singer: an exploration of holobiosis and evolutionary theory. Biol Philos 32(1):5-24

7. Bohnhoff M, Drake BL, Miller CP (1954) The effect of antibiotic therapy on susceptibility to an experimental enteric infection. Trans Assoc Am Physicians 67:156

8. van der Waaij D, Berghuis-de Vries JM, v Lekkerkerk Lekkerkerk (1971) Colonization resistance of the digestive tract in conventional and antibiotictreated mice. JHyg 69:405-411

9. Dethlefsen L, Relman DA (2011) Incomplete recovery and individualized responses of the human distal gut microbiota to repeated antibiotic perturbation. PNAS 108(Supplement 1):4554-4561

10. Becattini S, Littmann ER, Carter RA et al (2017) Commensal microbes provide first line defense against Listeria monocytogenes infection. J Exp Med 214:1973-1989

11. Zmora N, Zilberman-Schapira G, Suez J et al (2018) Personalized gut mucosal colonization resistance to empiric probiotics is associated with unique host and microbiome features. Cell 174:1388-1405

12. Litvak Y, Mon KKZ, Nguyen Het al (2019) Commensal Enterobacteriaceae protect against Salmonella colonization through oxygen competition. Cell Host Microbe 25:128-139
13. Iwase T, Uehara Y, Shinji H et al (2010) Staphylococcus epidermidis Esp inhibits Staphylococcus aureus biofilm formation and nasal colonization. Nature 465:346-349

14. Zipperer A, Konnerth M, Laux C et al (2016) Human commensals producing a novel antibiotic impair pathogen colonization. Nature 535:511-516

15. von Eiff C, Becker K, Machka K et al (2001) Nasal carriage as a source of Staphylococcus aureus bacteremia. NEngl J Med 344:11-16

16. Weidenmaier C, Goerke C, Wolz C (2012) Staphylococcus aureus determinants for nasal colonization. Trends Microbiol 20:243-250

17. Sugimoto S, Iwamoto T, Takada K et al (2013) Staphylococcus epidermidis Esp degrades specific proteins associated with Staphylococcus aureus biofilm formation and host-pathogen interaction. JBacteriol 195:1645-1655

18. Byrd A, Belkaid Y, Segre J (2018) The human skin microbiome. Nat Rev Microbiol 16:143-155

19. Bomar L, Brugger SD, Yost BH et al (2016) Corynebacterium accolens releases antipneumococcal free fatty acids from human nostril and skin surface triacylglycerols. mBio 7:e1725

20. Nakatsuji T, Chen TH, Narala S et al (2017) Antimicrobials from human skin commensal bacteria protect against Staphylococcus aureus and are deficient in atopic dermatitis. Sci Transl Med 9:eaah4680

21. Fraune S, Anton-Erxleben F, Augustin Ret al (2015) Bacteria-bacteria interactions within the microbiota of the ancestral metazoan Hydra contribute to fungal resistance. ISME J9(7):1543-1556

22. Zeeuwen PL, Kleerebezem M, Timmerman HM et al (2013) Microbiome and skin diseases. Curr Opin Allergy Clin Immunol 13(5):514-520

23. Finlay B, Amato KR, Azad Metal (2021) The hygiene hypothesis during a pandemic: consequences for the human microbiome. Proc Natl Acad Sci USA 118(6):e2010217118

24. Strachan DP (1989) Hay fever, hygiene, and household size. BMJ 299:1259-1260

25. Scudellari M (2017) News feature: cleaning up the hygiene hypothesis. Proc Natl Acad Sci USA 114:1433-1436 
26. Stiemsma LT, Reynolds LA, Turvey SE et al (2015) The hygiene hypothesis: current perspectives and future therapies. Immunotargets Ther 4:143-157

27. Sonnenburg JL, Sonnenburg ED (2019) Vulnerability of the industrialized microbiota. Science 366(6464):eaaw9255

28. Chiu FP, Doolan BJ, McGrath JA et al (2020) $A$ decade of next-generation sequencing in genodermatoses: the impact on gene discovery and clinical diagnostics. Br J Dermatol. https://doi. org $/ 10.1111 /$ bjd.19384

29. Finlay B, Pettersson S, Melby M, Bosch TCG (2019) The microbiome mediates environmental effects on ageing. Bioessays 41(10):e1800257. https://doi. org/10.1002/bies.201800257

30. Blaser MJ (2017) Antibiotika overkill. So entstehen die modernen Seuchen. Herder, Freiburg (Aus dem Englischen von Ulrich Magin)

\section{Entscheidungsassistenz}

Eine Brücke auf dem Weg zur Einwilligungsfähigkeit von Menschen mit Demenz in medizinische Maßnahmen

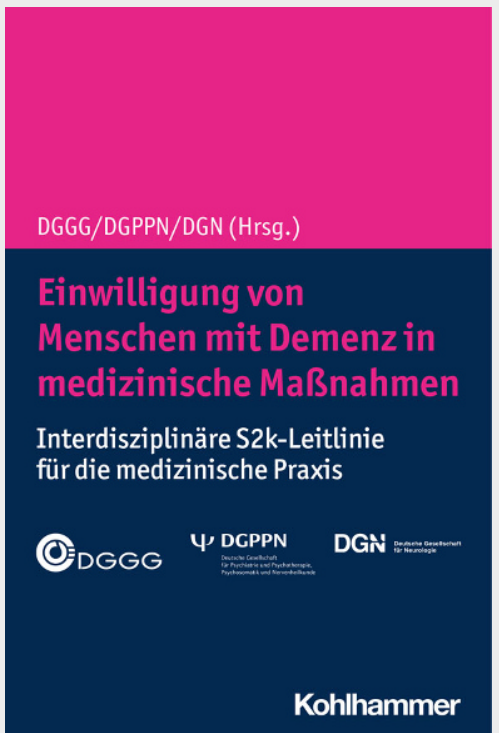

Die 2020 veröffentlichte Interdisziplinäre Leitlinie „Einwilligung von Menschen mit Demenz in medizinische Maßnahmen" [1] und die 2021 hierzu in der Zeitschrift für Gerontologie+Geriatrie CME-validierte Fortbildung [2] zeigen einen strukturierten Handlungspfad mit dem Ziel, die Selbstbestimmung des Menschen mit Demenz möglichst lange zu erhalten.

Die Diagnose einer Demenz kann und darf nicht mit Unfähigkeit zur Einwilligung gleichgesetzt werden. Die Entscheidung des Patienten für eine medizinische Maßnahme muss auf einer informierten Einwilligung beruhen. Die Voraussetzungen hierfür sind:

1. Informationsvermittlung

2. Informationsverständnis

3. freie Entscheidung

4. Einwilligungsfähigkeit

Bestehen Zweifel an der Einwilligungsfähigkeit und somit an der Bestimmbarkeit des Willens eines Menschen mit Demenz, muss die Einwilligungsfähigkeit im Sinne eines klinischen Urteils valide geprüft werden:

1. Informationsverständnis

2. Einsicht in die Krankheit und die Notwendigkeit der Behandlung

3. Urteilsvermögen

4. Kommunizieren einer Entscheidung

Die Synthese dieser vier Felder ergibt die

Bestimmbarkeit des Willens.
Erscheint die Einwilligungsfähigkeit des Gegenübers in einen medizinischen Eingriff nach valider Prüfung nicht oder nicht sicher gegeben, kann durch Herbeiführen einer so genannten Entscheidungsassistenz unter Umständen eine Einwilligungsfähigkeit erreicht werden.

Entscheidungsassistenz ließe sich als einen empathisch wertschätzenden individualisierten Prozess im Konzept des ärztlichen Aufklärungsgespräches in räumlicher, sozialer und dinglicher Kontextgestaltung definieren: eine ruhige Atmosphäre ohne störende Einflüsse, eine den Besonderheiten der Demenz und etwaigen weiteren Beeinträchtigungen der betreffenden Person angepasste Form der Kommunikation, vor allem das Sehen und das Hören betreffend, gegebenenfalls unter Hinzunahme notwendiger Hilfen und Hilfsmittel, ein klare und einfache bis hin zur leichten Sprache, bei Bedarf eine zusätzliche schriftliche Information, gegebenenfalls auch Anwesenheit einer Begleitperson. Fazit: Auch wenn nicht in allen Fällen bei betroffenen Personen eine Einwilligungsfähigkeit erreicht werden kann, so kann doch mithilfe dieses Konzeptes zwischen einer eher zustimmenden und einer eher ablehnenden Haltung („assent“ versus „dissent") differenziert werden.

\section{Literatur}

[1] DGGG/DGPPN/DGN (Hrsg.) (2020) Einwilligung von Menschen mit Demenz in medizinische Maßnahmen, Interdisziplinäre S2k-Leitlinie für die medizinische Praxis (AWMF-Leitlinie Registriernummer 108-001), 1. Auflage, W. Kohlhammer GmbH, Stuttgart; www.awmf.org/leitlinien/detail/ll/108001.html

[2] Haberstroh J, Tesky VA , Pantel J (2021) Einwilligungsfähigkeit von Menschen mit Demenz, Einblicke in die S2k-AWMF-Leitlinie 108-001.Z Gerontol Geriat 54:167-175; doi.org/10.1007/s00391-020-01820-4

\section{Korrespondenzadresse:}

Dr. Udo Hennighausen

Augenarzt, Anerkennung Geriatrie

Jürgen-Töpfer-Straße 10

22763 Hamburg

E-Mail: Udo. Hennighausen@web.de 